\title{
VEHICLE ROUTING PROBLEM WITH STOCHASTIC DEMANDS
}

In the introductory section of this paper there is a view of several types of stochastic vehicle routing problems. In the second section of the paper the vehicle routing problem with stochastic demands is described. The following modifications to the vehicle routing problem are required: Customers demand is a random variable with a known probability distribution. Routes must be designed before the actual demands become known.

The presence of nonlinear constraints, caused by random demands, can complicate the solution. Under the specific presumptions it is possible to transfer a nonlinear constraint to a linear form. This transformation enables to use the known algorithms of the deterministic vehicle routing problem for solution of the stochastic vehicle routing problem.

Keywords: Vehicle routing problem, stochastic demands, artificial capacity, programming system LINGO.

\section{Opening notes}

The classical vehicle routing problem $(V R P)$ is defined on a graph $G=(V, A)$, where $V=\left\{v_{1}, v_{2}, \ldots, v_{n}\right\}$ is a set of vertices and $A=\left\{\left(v_{i}, v_{j}\right): i \neq j, v_{i}, v_{j} \in V\right\}$ is the arcs set. Vertex $v_{1}$ represents a depot at which $m$ identical vehicles are based, while the remaining vertices correspond to customers. Matrix $C=\left(c_{i j}\right)$ is defined on a set $A$. The coefficients $c_{i j}$ represent distances or travel costs. The count of vehicles can be a given constant or a decision variable. All vehicles have the identical capacity $Q$. The $V R P$ is the problem of constructing $m$ vehicle routes of minimum total cost that each remaining vertex is visited exactly once by one vehicle and satisfying some side constraints.

Types of constraints:

Capacity constraints (each customer $v_{i}$ has a demand $d_{i}$ and the total demand of any route may not exceed the vehicle capacity).

Duration constraints (the total length of each route may not exceed a preset constant $L$ ).

Time window constraints (each vertex $v_{i}$ must be visited within a time interval $\left.\left[a_{i}, b_{i}\right]\right)$.

\section{Stochastic vehicle routing problem: An Introduction}

Stochastic vehicle routing problems $(S V R P)$ arise whenever some elements of the problem are random (e. g. stochastic demand and stochastic travel times).

SVRP differ from the deterministic VRP in several fundamental respects. The concept of a solution is different, solution methodologies are considerably more intricate. SVRP are often regarded as computationally intractable.
SVRP can be cast within the framework of stochastic programming. Stochastic programs are modeled in two stages. In the first stage a planned solution is determined. In the second stage corrective action is applied to the first stage solution.

Types of stochastic vehicle routing problems:

The vehicle routing problem with stochastic demand is without any doubt the most studied of all SVRP. Customer demands are random variables usually assumed to be independent of each other.

The vehicle routing problem with stochastic customers is a direct extension of the traveling salesman problem with stochastic customers. Each customer is represented with some probability but has deterministic demand. The vehicle capacity must be respected and return trips to depot may be necessary whenever it becomes attained.

The vehicle routing problem with stochastic customers and stochastic demands is a combination of the previous models. The definition proposed by Bertsimas [1] seems the most interesting. This vehicle routing problem is an exceedingly difficult problem.

In this paper we study an important variation of the $V R P$ in which customer demands are random variables. We consider the situation with unknown demand for each location, but is assumed to follow the known probability distribution.

\section{Stochastic vehicle routing problem with stochastic demands}

The SVRP is at least as difficult as the VRP. The presence of nonlinearities in the constraints caused by the probabilistic demands may, in fact, makes it considerably more difficult.

\footnotetext{
* Václav Kořenár̆

University of Economics Prague, Department of Econometrics, Praha 3, nám. W. Churchilla 4; E-mail: Korenar@vse.cz
} 
The following modifications [2] of the vehicle routing problem are required:

- customers demand is a random variable with a known probability distribution;

- routes must be designed before the actual demands become known.

If the demand at each customer location is a random variable, the problem becomes a stochastic vehicle routing problem and must be treated accordingly.

A mathematical formulation of the SVRP:

Minimize $\sum_{k} \sum_{i, j} c_{i j} x_{i j k}$

subject to

$$
\begin{aligned}
& \operatorname{Prob}\left\{\sum_{i, j} d_{i} x_{i j k} \leq Q\right\} \geq 1-\alpha \text { for } k=1,2, \ldots, m, \\
& x=\left[x_{i j k}\right] \in S,
\end{aligned}
$$

where $c_{i j}$ is the distance or cost of travelling from $i$ to $j, Q$ is the vehicle capacity, $m$ is the number of vehicles, $S$ is the set of all $m$ - feasible solutions, $x_{i j k}=1$ if $i$ and $j$ are joined on route $k, 0$ otherwise. The demands $d_{i}$ are random values of the known probability distribution. The parameter $\alpha$ is a probability that in proposed routes the total demand will be greater than the vehicle capacity. Constraint (2) is referred to a chance-constraint.

In the arguments that follow we demonstrate that a chance constraint can be transformed into an equivalent deterministic constraint. An artificial capacity will be used in a VRP algorithm in place of the true vehicle capacity. This artificial capacity will be less than the true capacity. Viewing mean customer demands as artificial demands and filling vehicles up to their artificial capacity will perform routing.

Consider a constraint

$$
\operatorname{Prob}\left\{\sum_{i} a_{i} y_{i} \leq b\right\} \geq 1-\alpha
$$

If the $a_{i}$ are independent and identically distributed random variables with the mean $\underline{a}_{i}$ and standard deviation $s_{i}$, and the $y_{i}$ are decision variables, then the mean is $M=\Sigma a_{i} y_{i}$ and the standard deviation is $S=\left(\sum s_{i}^{2} y_{i}^{2}\right)^{1 / 2}$. We seek the constant $T$ such that

$$
\operatorname{Prob}\left\{\left(\sum_{i} a_{i} y_{i}-M\right) / S \leq T\right\}=1-\alpha .
$$

If $b \geq M+T S$, then the left-hand side of inequality (3) is also not less than $1-\alpha$. Constraint (3) can be replaced by the deterministic constraint

$$
M+T S \leq b .
$$

Constraint (5) is nonlinear. When we rewrite this constraint in terms of $y_{i}$, we obtain

$$
\sum_{i} \underline{a}_{i} y_{i}+T\left(\sum_{i} s_{i}^{2} y_{i}^{2}\right)^{1 / 2} \leq b
$$

The presence of nonlinear constraints caused by the random demands can complicate the solution. Under the specific presumptions it is possible to transfer a nonlinear constraint (6) to a linear constraint. We note that in the context of the SVRP the $a_{i}$ is probabilistic demand and the $y_{i}$ is $0-1$ variable. If $M$ is a mean of customer random demand, $S^{2}$ is a variance of random demand and if $S^{2}=\lambda M$ for some constant $\lambda$, we can transfer a nonlinear constraint (6) to a linear form as

$$
\sum_{i} \underline{a}_{i} y_{i}+T\left(\lambda \sum_{i} \underline{a}_{i} y_{i}\right)^{1 / 2} \leq b
$$

or

$$
M+T \sqrt{ }(\lambda M) \leq b
$$

We intend to modify this inequality in the simple form

$$
M \leq \underline{b} .
$$

Define $w=T \sqrt{ }$, then we obtain from (8)

$b-M \geq w M^{1 / 2}$

or

$(b-M)^{2} \geq w^{2} M$.

It follows from (10)

$$
M \leq\left[2 b+w^{2}-\sqrt{ }\left(w^{4}+4 b w^{2}\right)\right] / 2=\underline{b} .
$$

If $T$ is determined by $\operatorname{Prob}(z \leq T)=1-\alpha$, where $z$ is standard normal distributed, we get from (11) the artificial capacity $\underline{b}$. The expression for the artificial capacity like (11) can be formulated also in case of gamma, binomial and Poisson distributions.

\section{Illustrative example}

As an illustration we solve the vehicle routing problem with random demand of customers, which has Poisson distribution. An expected value of Poisson distribution is equal to its variance and so we can use (11) to compute artificial capacity. And it is satisfied $w=T$, because $\lambda=1$ for Poisson distribution. Parameter $T$ can be found for a given value $\alpha$ as an quantile of normal distributions in statistical tables.

Let suppose that the true capacity of a vehicle is equal to 30 . If we compute (11) with our $w$ and $b$, we get the artificial capacity and solve the vehicle routing problem as deterministic. Instead of the true capacity vehicles we use the artificial one.

The results of the calculation of artificial capacity $\underline{b}$ using (11) can be found in Table 1. 
Table 1

Table 2

\begin{tabular}{|c|c|c|}
\hline$\alpha$ & $\mathrm{T}$ & $\underline{b}$ \\
\hline 0.9 & 1.282 & 23.75 \\
\hline 0.95 & 1.645 & 22.24 \\
\hline 0.99 & 2.326 & 19.68 \\
\hline
\end{tabular}

We solved the vehicle routing problem with 10 locations by the system LINGO using branch and bound algorithm.

Summary results are in Table 2:

\begin{tabular}{|l|c|c|c|c|}
\hline Vehicle capacity & Capacity & $\begin{array}{c}\text { Time of } \\
\text { calculation } \\
(\mathrm{min})\end{array}$ & $\begin{array}{c}\text { Goal } \\
\text { function }\end{array}$ & $\begin{array}{c}\text { Number } \\
\text { of } \\
\text { iterations }\end{array}$ \\
\hline \multicolumn{1}{|c|}{ True capacity } & 30 & $2: 08$ & 114 & 223684 \\
\hline $\begin{array}{l}\text { Artificial capacity } \\
(\alpha=0,9)\end{array}$ & 23,75 & $2: 42$ & 117 & 283662 \\
\hline $\begin{array}{l}\text { Artificial capacity } \\
(\alpha=0,95)\end{array}$ & 22,24 & $4: 34$ & 122 & 478925 \\
\hline $\begin{array}{l}\text { Artificial capacity } \\
(\alpha=0,99)\end{array}$ & 19,68 & $8: 00$ & 127 & 868025 \\
\hline
\end{tabular}

\section{References}

[1] BERTSIMAS, D. J.: A Vehicle Routing Problem with Stochastic Demand. Operations Research, vol. 40, 1992, pp. 574-585.

[2] BODIN, L., GOLDEN, B., ASSAD, A., BELL, M.: Routing and Scheduling of Vehicles and Crews. State of Art. Comput. \& Ops. Res., Vol. 10, No 2, 1983, pp. 109-111.

[3] GENDREAU, M., LAPORTE, G., SÉGUIN, R.: Stochastic Vehicle Routing. European Journal of Operational Res., Vol. 88, 1996, pp. 3-12.

[4] KOŘENÁŘ, V., VÍŠEK, T.: Branch and Cut Algorithm for Routing Problem. In: Plevný, M., Friedrich, V. (ed.). Mathematical Methods in Economics. Plzeň, Západočeská univerzita, 1998, s. 65-72. ISBN 80-7082-492-1.

[5] Solver Suite - LINDO, LINGO, What's Best. Lindo System, Chicago 1995. 